\title{
Improving Patient Care, Medical Education, and Biomedical Research in the United States: The Role of Social Media
}

\section{Timothy L Wiemken* and Robert R Kelley}

Division of Infectious Diseases, University of Louisville, Louisville, KY, USA

\section{The Healthcare System}

The healthcare system is one of the largest and most important multinational enterprises in the United States [1]. Healthcare service is comprised of three essential activities including patient-care, education, and research. In 2008, over 14 million residents in the United States were employed in the healthcare field, excluding related industries such as insurance and pharmaceutical companies [2]. The United States spends roughly $\$ 8,000$ per person, per year on healthcare (a growing figure), which is $51 \%$ more than the nation with the next largest expenditure [3]. A significant amount of this spending is on health research activities (e.g. National Institutes of Health, Centers for Disease Control and Prevention). Although this spending is a noteworthy share of the Nation's Gross Domestic Product, it can be valuable if products are produced that can put this money back into the system. The development of new knowledge and products via healthcare research activities is one viable method of replacing this capital.

\section{Problems with the System}

The three essential service activities in healthcare have historically operated in separate silos with limited collaboration within or between them. This significantly reduces the efficiency of the system, impeding the development of new knowledge and marketable intellectual property.

\section{Patient-care}

In the area of patient care, collaboration between clinical services and other departments has been limited. The majority of information transfer is done via the medical record, which often times may be illegible or poorly documented. A lack of efficient and effective information transfer may lead to inadequate care and/or poor patient outcomes.

\section{Education}

The paradigm utilized for education has not changed since the middle Ages. Separate schools, departments, and divisions largely focus on their own programs, with little communication or collaboration between them. Redundant and outdated information are often provided to students under this paradigm, which may delay the student's ability to acquire best and most up-to-date information.

\section{Research}

Healthcare research suffers from similar issues as patient care and education. The time has passed when the lone scientist could succeed in the creation of new knowledge. In today's research environment, efficient collaboration between a diverse set of physicians and scientists is necessary. Without such collaboration, less than half of the breakthroughs in basic science will be developed into products that can influence patient care [4]. Unfortunately, this statistic has been a reality for years, and will not change without a radical paradigm shift. Furthermore, the current lack of translation impedes healthcare economic development and the translation of basic research results into clinical and public health practice. This increases healthcare costs and decreases the quality of healthcare.

\section{The Role of Social Media}

Currently, few studies suggest potential remedies for collaboration issues in patient care and medical education. In the area of research however, the National Institutes of Health recently reformulated its research agenda and created a new model known as Clinical and Translational Science [5]. This new paradigm recognizes the need to increase collaboration that could pave the way for the translation of basic science findings into products that can benefit patient care and public health. This model proposes several unique views that may be effective for the reformulation of patient care and educational activities.

While implementing a new model to increase collaboration is not simple, it is certainly possible. Our group has recently developed a successful program in translational science, encompassing each of the three elements of healthcare [6]. Regardless of these activities, adopting this paradigm only provides the "why" and not the "how" with regard to implementation of new communication and collaborations strategies.

Recently, authors have suggested that social media and social networks can provide the answer to "how" [7-9]. Dynamic web and mobile device applications deliver the ability to maintain real-time communication and up-to-date information exchange between multiple parties. Online social networking applications such as Facebook and Twitter are ideal for translational science collaboration, as they can help to not only maintain communication in both centralized and decentralized networks, but may also help to forge new and diverse contacts. Video conferencing software (e.g. Skype or FaceTime) allows on-demand, face-to-face communication between collaborators locally, nationally, and internationally. Moreover, video sharing services such as YouTube provide opportunities for skills-based information transfer, which have been previously limited by geography and our traditional model of unidirectional information transfer. All

*Corresponding author: Timothy Wiemken, Division of Infectious Diseases University of Louisville, Louisville, KY, USA, Tel: +502-852-4627; E-mail: tim.wiemken@louisville.edu

Received February 01, 2012; Accepted February 06, 2012; Published February 10,2012

Citation: Wiemken TL, Kelley RR (2012) Improving Patient Care, Medical Education, and Biomedical Research in the United States: The Role of Social Media. J Socialomics 1:e106. doi:10.4172/2167-0358.1000e106

Copyright: (c) 2012 Wiemken TL, et al. This is an open-access article distributed under the terms of the Creative Commons Attribution License, which permits unrestricted use, distribution, and reproduction in any medium, provided the original author and source are credited. 
Citation: Wiemken TL, Kelley RR (2012) Improving Patient Care, Medical Education, and Biomedical Research in the United States: The Role of Social Media. J Socialomics 1:e106. doi:10.4172/2167-0358.1000e106

Page 2 of 2

of these technologies are further supported through mobile devices like smart phones and tablet computers, which are pervasive in our society. With these technologies, physicians are able to communicate more efficiently than through the antiquated medical record, students have immediate access to the most current health-related information and skills, and investigators are able to collaborate in ways beyond the traditional face-to-face meeting.

Although technology continues to advance, the healthcare infrastructure has not kept pace. In order to allow physicians, students, and researchers to efficiently collaborate, the system must adapt. Medical facilities must fund information technology and infrastructure that will benefit the system over time, even if that means an increase in short-term costs. Like many businesses, healthcare organizations must support the tools and devices that are utilized by physicians, students, and investigators. For example, if a group sought to perform disease surveillance through social media, it would be difficult if those websites were blocked by the firewall at the perimeter of the network. At the end of the day, for the healthcare system to advance, it must embrace technology in an open fashion.

\section{Conclusions}

It is clear that our healthcare system is flawed due to barriers in communication and collaboration. The extravagant spending and poor patient outcomes seen in the United States are at least partly due to the limited development of new products resulting from a lack of collaboration across patient care, education and research. Technological advances such as mobile devices and social media may help eliminate these barriers, but must be adequately supported by the system to be effective. Embracing technology will increase communication and collaboration across each essential service within the healthcare system, and has great potential to decrease healthcare costs and increase the quality of care provided in the United States.

\section{References}

1. Jasso-Aguilar R, Waitzkin $\mathrm{H}$, Landwehr A (2004) Multinational corporations and health care in the United States and Latin America: strategies, actions, and effects. J Health Soc Behav 45: 136-157.

2. Bureau of Labor Statistics US Department of Labor (2012) Career Guide to Industries, 2010-11 Edition.

3. The Henry J Kaiser Family Foundation (2011) Healthcare spending in the United States and selected OECD countries.

4. Woolf SH (2008) The meaning of translational research and why it matters. JAMA 299: 211-213.

5. National Institutes of Health (2011) The NIH Common Fund.

6. Wiemken T, Peyrani P, Carrico R, Kelley R, Heragu S, et al. The Influenza Clinical and Research Program Investigators. Clinical and translational research in influenza at the University of Louisville. Journal of the Kentucky Medical Association.

7. George DR, Dellasega C (2011) Social media in medical education: two innovative pilot studies. Med Educ 45: 1158-1159.

8. Hunt JD, Whipple EC, McGowan JJ (2012) Use of social network analysis tools to validate a resources infrastructure for interinstitutional translational research: a case study. J Med Libr Assoc 100: 48-54.

9. Ruttenberg A, Clark T, Bug W, Samwald M, Bodenreider O, et al. (2007) Advancing translational research with the Semantic Web. BMC Bioinformatics 8: S2. 